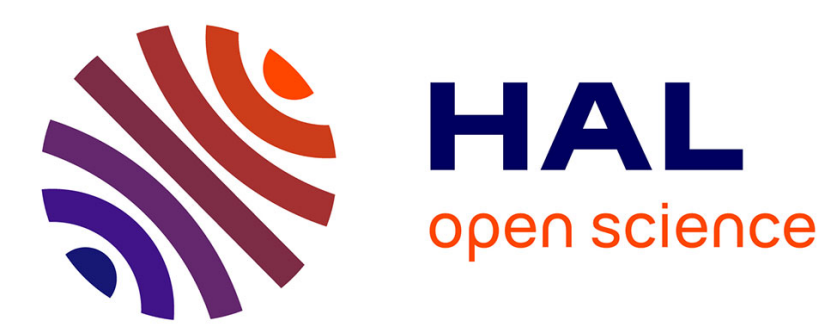

\title{
Asymptotic Performance for Delayed Exponential Process
}

\author{
Remy Boyer, Karim Abed-Meraim
}

\section{To cite this version:}

Remy Boyer, Karim Abed-Meraim. Asymptotic Performance for Delayed Exponential Process. IEEE Transactions on Signal Processing, 2007, 55 (6). hal-00575668

\section{HAL Id: hal-00575668 \\ https://hal.science/hal-00575668}

Submitted on 10 Mar 2011

HAL is a multi-disciplinary open access archive for the deposit and dissemination of scientific research documents, whether they are published or not. The documents may come from teaching and research institutions in France or abroad, or from public or private research centers.
L'archive ouverte pluridisciplinaire HAL, est destinée au dépôt et à la diffusion de documents scientifiques de niveau recherche, publiés ou non, émanant des établissements d'enseignement et de recherche français ou étrangers, des laboratoires publics ou privés. 


\section{Correspondence}

\section{Asymptotic Performance for Delayed Exponential Process}

Rémy Boyer and Karim Abed-Meraim

\begin{abstract}
The damped and delayed sinusoidal (DDS) model can be defined as the sum of $M$ sinusoids whose waveforms can be damped and delayed. This model is suitable for compactly modeling short time events. This property is closely related to its ability to reduce the time-support of each sinusoidal component. In this correspondence, we derive exact and approximate asymptotic Cramér-Rao bounds (CRBs) for the DDS model. This analysis shows that this model has better, or at least similar, theoretical performance than the well-known exponentially damped sinusoidal (EDS) model. In particular, the performance in the DDS case is significantly improved compared to that of the EDS for closely spaced sinusoids thanks to the nonzero time delays. Consequently, we can exploit the advantageous properties of the DDS model and, in the same time, we can keep high theoretical model parameter estimation accuracy.
\end{abstract}

Index Terms-Approximate bound, conditional Cramér-Rao bound (CCRB), delayed sinusoids.

\section{INTRODUCTION}

Parametric models such as the constant-amplitude sinusoidal or exponentially damped sinusoidal (EDS) models are popular and efficient tools in many areas of interest including pole estimation [1], source localization [2], biomedical signal processing [4], and audio signal compression [6]. In this correspondence, we use a generalization of these models, named the damped and delayed sinusoidal (DDS) model which adds a time-delay parameter to allow time-shifting of each component waveform [5], [3]. Even though the question of the design of model parameter estimation algorithms has been treated in [3] and [5], the asymptotic performance of this model has not been completely studied.

The contribution of this correspondence is the derivation and the comparison of several conditional Cramér-Rao bound (CCRB). This bound allows the analysis of the achievable theoretical performance of the DDS model in the situation where we exactly know the time-delay parameters. Note that a numerical bound has already been proposed in a companion paper [3], but here we go further into the asymptotic performance analysis of this model.

More specifically, we present the derivation of the exact CCRB. Next, to highlight the time-delay dependency of this bound in the context of large time-spacing between two consecutive DDS waveforms, we introduce an asymptotic approximate conditional CRB (ACCRB) which is shown to be insensitive to the time-delay values. It is important to note that no restricting assumption on the damping-factor value is made and we allow in our analysis overlapping consecutive waveforms. To further analyze the asymptotic performance of this model, we compare the ACCRB for the DDS model to the CRB for the EDS model. We

Manuscript received September 12, 2005; revised August 20, 2006. The associate editor coordinating the review of this manuscript and approving it for publication was Dr. Lang Tong.

R. Boyer is with the Université Paris XI (UPS), CNRS-LSS-Supélec, 91190 Gif-sur-Yvette, France (e-mail: remy.boyer@1ss.supelec.fr).

K. Abed-Meraim is with the Laboratoire Traitement du Signal et des Images, GET-ENST, 75634 Paris Cedex 13, France (e-mail: abed@tsi.enst.fr).

Color versions of one or more of the figures in this paper are available online at http://ieeexplore.iee.org.

Digital Object Identifier 10.1109/TSP.2007.893980 show that the distance between the CRB and the ACCRB is small for well-separated angular frequencies and very large for closely spaced poles. The latter case can be explained by the fact that the Fisher information matrix (FIM) CCRB for the DDS model remains low. Consequently, the DDS model shows at least similar theoretical performance as the model without time delay (i.e., the EDS model). In other words, it becomes possible to exploit the advantage of the DDS model and at the same time one can keep high theoretical performance.

\section{DDS MODEL}

The complex $M$-DDS model [3], [5] definition is given by

$$
\hat{x}(n)=\sum_{m=1}^{M} a_{m} e^{i \phi_{m}} e^{\left(i \omega_{m}+d_{m}\right)\left(n-t_{m}\right)} \psi\left(n-t_{m}\right)
$$

where $M$ is the number of complex sinusoids and $\left\{a_{m}, \phi_{m}, d_{m}, \omega_{m}, t_{m}\right\}_{1 \leq m \leq M}$ are (nonzero) real amplitude, phase, damping factor, and angular frequency parameters. We denote the $m$ th pole by $z_{m}=e^{i \omega_{m}+d_{m}}$ and we assume that all the angular frequencies are distinct: $\omega_{i} \neq \omega_{j}$ for $i \neq j, \omega_{i} \in(0, \pi]$ and $d_{i}<0$. In (1), we have introduced the discrete-valued time-delay parameters $\left\{t_{m}\right\}$ and the Heaviside function defined by $\psi(n)=1$ for $n \geq 0$, and 0 otherwise.

\section{CCRB FOR THE DDS MODEL}

The Cramér-Rao bound (CRB) is useful as a touchstone against which the efficiency of the considered estimators can be tested. Consider an $M$-DDS process corrupted by zero-mean white Gaussian noise $w(n)$ according to

$$
x(n)=\hat{x}(n)+\sigma w(n), \quad n \in[0: N-1]
$$

where $\hat{x}(n)$ is given by (1). Let $\Omega=\left[\begin{array}{lll}\omega_{1} \cdots \omega_{M} & d_{1} \cdots d_{M}\end{array}\right]^{T}$ (respectively, $\Upsilon=\left[\begin{array}{lll}\Omega^{T} & \sigma^{2} & t_{1} \cdots t_{M}\end{array}\right]^{T}$ with $\left.t_{1} \leq \cdots \leq t_{M}\right)$ be the vector of desired (respectively, desired plus nuisance) model parameters. Note that in the following, we assume for simplicity that the complex amplitude are known, and therefore, we omit this parameter in the derivation of our bounds.

The CRB, which is given by the diagonal terms of the FIM inverse [7], is a lower bound on the variance of the model parameters, i.e., $\operatorname{MSE}(\Upsilon) \geq \operatorname{CRB}(\Upsilon)=F_{\Upsilon}^{-1}$ where $F_{\Upsilon}$ denotes the FIM for parameter $\Upsilon$ and MSE stands for mean-squared error.

Note that the time delay has discrete value and is considered as perfectly known; so in the sequel, this parameter will be omitted and we name this new bound CCRB for conditional CRB. Moreover, we can formulate Property 1 (the proof is provided in [3]).

Property 1: The elements of the FIM corresponding to the cross terms of $\Omega_{i}$ and $\sigma^{2}$ are zero.

In other words, the CCRB for $\Omega$ is decoupled from the CCRB for $\sigma^{2}$; we can also omit the noise variance in the computation of the CCRB. Consequently, we retain only vector $\Omega$ to derive the CCRB. Its definition is given according to

$$
\begin{aligned}
\operatorname{CCRB}(\Omega \mid t) & =F_{\Omega}^{-1} \text { with }\left[F_{\Omega}\right]_{i, j} \\
& =E\left[\frac{\partial \mathcal{L}_{t}(x \mid \Omega)}{\partial \Omega_{i}}\left(\frac{\partial \mathcal{L}_{t}(x \mid \Omega)}{\partial \Omega_{j}}\right)^{H}\right]
\end{aligned}
$$


where $E[\cdot]$ is the mathematical expectation. Under those assumptions, the logarithmic likelihood function can be expressed as $\mathcal{L}_{t}(x \mid \Omega)=$ $c-N \log \sigma^{2}-|x-\hat{x}|^{2} \sigma^{2}$ where $c$ is a given constant.

Theorem 1: The CCRB for the variance of any unbiased estimate of $\Omega$ (conditionally to the perfect knowledge of the time-delay parameter vector $t$ ) is given by

$$
\operatorname{CCRB}(\Omega \mid t)=F_{\Omega}^{-1} \quad \text { where } F_{\Omega}=\frac{2}{\sigma^{2}} \Re e\left\{\frac{\partial \hat{x}}{\partial \Omega}\left(\frac{\partial \hat{x}}{\partial \Omega}\right)^{H}\right\} .
$$

$\mathfrak{R e}(\cdot)$ is the real part of a complex entity.

In other words, the FIM is proportional to the inverse of the derivative matrix. Following the same methodology as in [8], we obtain the expression of the CCRB according to

$$
\operatorname{CCRB}(\Omega \mid t)=\frac{\sigma^{2}}{2}\left(I_{2} \otimes A^{-1}\right) Q^{-1}\left(I_{2} \otimes A^{-1}\right)
$$

where $Q=\Re e\left\{P P^{H}\right\}, A=\operatorname{diag}\left\{a_{1}, \cdots, a_{M}\right\}$ and $P=\left[\begin{array}{c}i \Phi P_{N} \\ \Phi P_{N}\end{array}\right]$ with $\Phi=\operatorname{diag}\left\{e^{i \phi_{1}}, \cdots, e^{i \phi_{M}}\right\}$ and

$$
P_{N}=\left[\begin{array}{cccc}
0_{t_{1}+1} & z_{1} & \cdots & \left(N-t_{1}-1\right) z_{1}^{N-t_{1}-1} \\
0_{t_{2}+1} & z_{2} & \cdots & \left(N-t_{2}-1\right) z_{2}^{N-t_{2}-1} \\
\vdots & \vdots & & \vdots \\
0_{t_{M}+1} & z_{M} & \cdots & \left(N-t_{M}-1\right) z_{M}^{N-t_{M}-1}
\end{array}\right] .
$$

We begin by the following derivation. Define matrix $G$ which $(i, j)$ th entry is

$$
\begin{aligned}
g_{i j}= & {\left[\Phi P_{N} P_{N}^{H} \Phi^{*}\right]_{i j} } \\
= & e^{i\left(\phi_{i}-\phi_{j}\right)} z_{i}^{-t_{i}} z_{j}^{-t_{j}} \sum_{n=0}^{N-1}\left(n-t_{i}\right)\left(n-t_{j}\right) \\
& \times\left(z_{i} z_{j}^{*}\right)^{n} \psi\left(n-t_{i}\right) \psi\left(n-t_{j}\right)
\end{aligned}
$$

and $Q=\mathfrak{R} e\left\{\left[\begin{array}{cc}G & i G \\ -i G & G\end{array}\right]\right\}$. Without loss of generality, we sort the time delays according to $\tau_{i j}=t_{j}-t_{i} \geq 0$ for $j \geq i$. Since $\psi(n-$ $\left.t_{i}\right) \psi\left(n-t_{j}\right)=\psi\left(n-t_{j}\right)$, (8) becomes for $j \geq i$

$g_{i j}=z_{i}^{\tau_{i j}} e^{i\left(\phi_{i}-\phi_{j}\right)}\left(\sum_{n=0}^{N-t_{j}-1} n^{2}\left(z_{i} z_{j}^{*}\right)^{n}+\tau_{i j} \sum_{n=0}^{N-t_{j}-1} n\left(z_{i} z_{j}^{*}\right)^{n}\right)$

where we explicit the sums in the previous expression according to

$$
\begin{aligned}
& \sum_{n=0}^{N-t_{j}-1} n^{2}\left(z_{i} z_{j}^{*}\right)^{n} \\
& =-\frac{\left(N-t_{j}-1\right)^{2}\left(z_{i} z_{j}^{*}\right)^{N-t_{j}-1}}{1-z_{i} z_{j}^{*}} \\
& \quad+\frac{1-\left(z_{i} z_{j}^{*}\right)^{N-t_{j}-1}-2\left(N-t_{j}-1\right)\left(z_{i} z_{j}^{*}\right)^{N-t_{j}-1}}{\left(1-z_{i} z_{j}^{*}\right)^{2}} z_{i} z_{j}^{*} \\
& \quad+\frac{2\left(z_{i} z_{j}^{*}\right)^{2}\left(1-\left(z_{i} z_{j}^{*}\right)^{N-t_{j}-1}\right)}{\left(1-z_{i} z_{j}^{*}\right)^{3}} \\
& \sum_{n=0}^{N-t_{j}-1} n\left(z_{i} z_{j}^{*}\right)^{n} \\
& =z_{i} z_{j}^{*} \frac{1-\left(z_{i} z_{j}^{*}\right)^{N-t_{j}-1}}{\left(1-z_{i} z_{j}^{*}\right)^{2}}-\frac{\left(N-t_{j}-1\right)\left(z_{i} z_{j}^{*}\right)^{N-t_{j}-1}}{1-z_{i} z_{j}^{*}} .
\end{aligned}
$$

The exact computation of the CCRB is given by (5)-(9). To obtain more tractable formulation, we consider the asymptotic CCRB.

\section{ASYMPTOTIC AND APPROXIMATE CCRB}

We make the following three assumptions.

A1) $N$ is sufficiently large according to $N \gg t_{M}$.

A2) $\left|d_{i}\right| \ll 1$ for $i \in[1: M]$. Indeed, the case where $\left|d_{i}\right|$ is large is not really of interest as it corresponds to the situation where the components have short time supports, and hence, they are well separated, in which case the CRB would be close to that of a monocomponent signal.

A3) The duration between two consecutive waveforms has to be sufficiently large, i.e., $\tau_{i, i+1} \gg 1$. However, due to A2), the $i$ th waveform can have a time support much larger than $\tau_{i, i+1}$, and thus, overlapping waveforms are possible.

\section{A. Asymptotic Expression of the CCRB}

Based on A1), expression (9) can be simplified according to

$$
g_{i j} \stackrel{N \rightarrow \infty}{\longrightarrow} g_{i j}^{(\infty)}=z_{i}^{\tau_{i j}} \xi_{i j}^{(1)}\left(\tau_{i j}+\xi_{i j}^{(2)}\right) e^{i\left(\phi_{i}-\phi_{j}\right)}
$$

where

$$
\xi_{i j}^{(1)} \stackrel{\text { def }}{=} \frac{z_{i} z_{j}^{*}}{\left(1-z_{i} z_{j}^{*}\right)^{2}} \quad \xi_{i j}^{(2)} \stackrel{\text { def }}{=} \frac{1+z_{i} z_{j}^{*}}{1-z_{i} z_{j}^{*}} .
$$

Note that $\xi_{i j}^{(1)}$ and $\xi_{i j}^{(2)}$ are two complex quantities independent of the time delays. In addition, thanks to A2) which allows us to consider a first-order approximation (using long division of Taylor series), the diagonal terms of matrix $G$ are real, independent of the time delays, the angular frequencies, and the phases since

$$
g_{i i}^{(\infty)}=\xi_{i i}^{(1)} \xi_{i i}^{(2)}=\frac{1}{4 d_{i}^{2}}\left(\frac{-1}{d_{i}}\right)=\frac{-1}{4 d_{i}^{3}} .
$$

We will distinguish here two cases. The first one is for well-separated poles and the second is for closely spaced poles.

\section{B. Well-Separated Poles}

Assume that the poles $z_{i}$ and $z_{j}$ are well separated according to

$$
\left|\omega_{i j}\right| \gg\left|d_{i}\right|^{b}
$$

where $b$ is a positive value specified in the sequel and $\omega_{i j}=\omega_{i}-\omega_{j}$. Under (13), the expression of $\left|g_{i j}^{(\infty)}\right|$ for $j>i$ can be upper-bounded as follows:

$$
\begin{aligned}
\left|g_{i j}^{(\infty)}\right| & =\left|z_{i}^{\tau_{i j}} \xi_{i j}^{(1)}\left(\tau_{i j}+\xi_{i j}^{(2)}\right)\right| \\
& \leq \frac{\left|z_{i}^{\tau_{i j}}\right|\left|\tau_{i j}\left(1-z_{i} z_{j}^{*}\right)+\left(1+z_{i} z_{j}^{*}\right)\right|}{\left|1-e^{i \omega_{i j}+d_{i}+d_{j}}\right|^{3}} \\
& \approx \frac{\left|z_{i}^{\tau_{i j}}\right|\left|\tau_{i j}\left(1-z_{i} z_{j}^{*}\right)+\left(1+z_{i} z_{j}^{*}\right)\right|}{\mid 1-e^{\left.i \omega_{i j}\right|^{3}}} \\
& \leq \frac{e^{d_{i} \tau_{i j}}\left(\tau_{i j}+2\right)}{\frac{8}{\pi^{3}}\left|\omega_{i j}\right|^{3}} \\
& \leq \frac{-1}{e d_{i} \frac{8}{\pi^{3}}\left|\omega_{i j}\right|} .
\end{aligned}
$$

In those inequalities, we have used the facts that $\left|1-e^{i \omega_{i j}+d_{i}+d_{j}}\right| \approx$ $\left|1-e^{i \omega_{i j}}\right|=\left|2 \sin \omega_{i j} / 2\right| \geq 2 \pi\left|\omega_{i j}\right|$ and the minimal value of the 
function $e^{d x}(x+2)$ is approximately equal to $-1 /(e d)$. By comparing $\left|g_{i j}^{(\infty)}\right|$ to $\left|g_{i i}^{(\infty)}\right|$, one can observe

$$
\frac{\left|g_{i j}^{(\infty)}\right|}{\left|g_{i i}^{(\infty)}\right|} \leq \frac{\pi^{3}}{2 e} \frac{d_{i}^{2}}{\left|\omega_{i j}\right|^{3}}
$$

Hence, by choosing $b=2 / 3$ in (13), we guarantee that the previous ratio is negligible, i.e., $\left|g_{i j}^{(\infty)}\right| /\left|g_{i i}^{(\infty)}\right| \ll 1$, and hence, in that case, one can approximate the CCRB by

$$
\begin{aligned}
& \operatorname{CCRB}(\Omega \mid t) \longrightarrow \operatorname{ACCRB}(\Omega) \stackrel{\text { def }}{=} \mathbb{F}^{-1} \\
& =\frac{\sigma^{2}}{2}\left(I_{2} \otimes A^{-1}\right) \mathbb{Q}^{-1}\left(I_{2} \otimes A^{-1}\right) \\
& =\frac{\sigma^{2}}{2} I_{2} \otimes(A G A)^{-1}
\end{aligned}
$$

where $\mathbb{Q}=I_{2} \otimes \mathbb{G}$ with $\mathbb{G}=\operatorname{diag}\left\{g_{11}^{(\infty)}, \cdots, g_{M M}^{(\infty)}\right\}$. Based on these considerations, we have Theorem 2 .

Theorem 2: Under A1)-A3) and condition (13), a closed form of the ACCRB with respect to the model parameters is given by

$$
\operatorname{ACCRB}\left(\Omega_{i}\right)=-2 \sigma^{2} \frac{d_{i}^{3}}{a_{i}^{2}}, \quad i \in[1: M] .
$$

Following Theorem 2, the ACCRB is invariant with respect to the phase, angular frequency, and time-delay parameter; so, the asymptotic performance is relied only to the length of each waveform (i.e., damping factors and amplitudes) and not to its oscillatory character nor to its time delay. Note that in standard Fourier analysis, the poles are considered well separated when $\left|\omega_{i j}\right| \gg 1 / N, N$ being the sample size. Condition (13) has similar meaning as for a small damping factor $d_{i}$ the effective sample size $N$ is of order $1 / d_{i}$, and hence (13) can be translated into $\left|\omega_{i j}\right| \gg 1 / N^{2 / 3}$.

\section{Identical Poles}

We consider now the case where two poles are equal, i.e., $z_{i}=z_{j}$. This is a limit case that illustrates the situation of closely spaced poles. In this context, the FIM is not anymore approximately diagonal but block-diagonal with diagonal blocks given by

$$
G_{i j}=\left[\begin{array}{cc}
g_{i i}^{(\infty)} & g_{i j}^{(\infty)} \\
g_{i j}^{(\infty) *} & g_{j j}^{(\infty)}
\end{array}\right] .
$$

The eigenvalues coincide with the roots of the characteristic polynomial

$\lambda^{2}-\operatorname{Tr}\left(G_{i j}\right) \lambda+\operatorname{det}\left(G_{i j}\right) \approx \lambda^{2}+\frac{1}{2 d_{i}^{3}} \lambda+\frac{1-e^{2 d_{i} \tau_{i j}}\left(d_{i} \tau_{i j}+1\right)^{2}}{16 d_{i}^{6}}$

which are approximately $\lambda_{ \pm}=\left(-1 /\left(4 d_{i}^{3}\right)\right)\left(1 \pm e^{d_{i} \tau_{i j}}\left(d_{i} \tau_{i j}+1\right)\right)$. Clearly, when $\tau_{i j} \gg 1$, the minimum eigenvalue $\lambda_{-}$is far from zero, while for $\tau_{i j}=0$, this eigenvalue becomes null. This latter situation represents the one of the EDS model where closely spaced sinusoids lead to significant performance degradation, i.e., in that case, the FIM becomes close to singular. However, thanks to the approximate blockdiagonal structure of the FIM, one can observe that the bad estimation of closely spaced poles does not affect much the estimation of the other poles.

\section{COMPARISON TO THE EDS MODEL}

In this section, we compare the DDS and the EDS models in the case of strong damping factor, i.e., $\left|d_{i}\right|=O(1)$ and in the case of well-separated poles with $\left|d_{i}\right| \ll 1$ for $i \in[1: M]$. Note that we have already shown that for closely spaced sinusoids the DDS significantly outperforms the EDS model. (a)

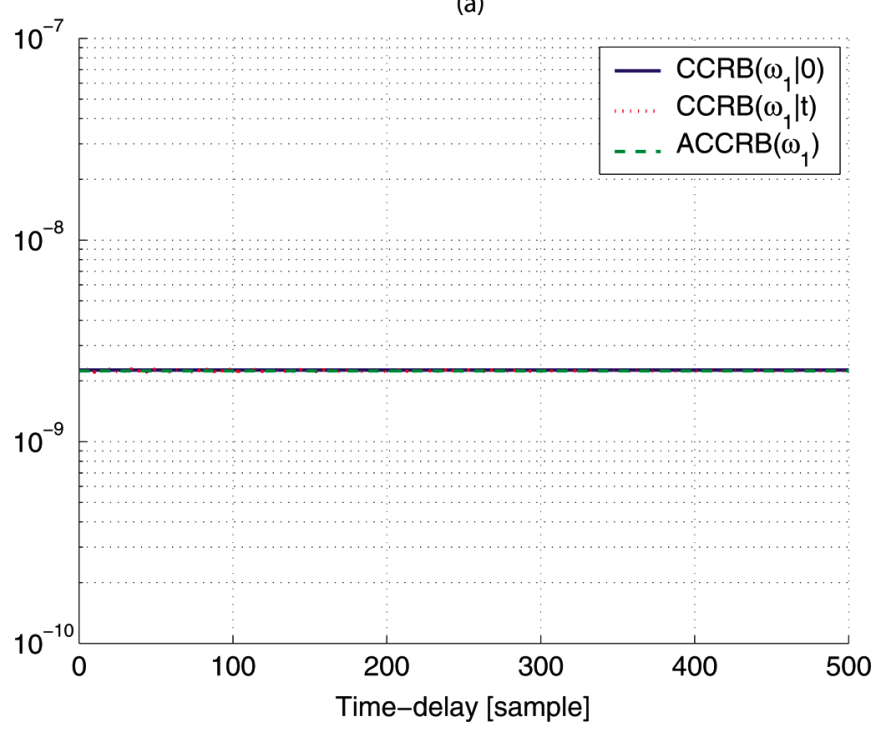

(b)

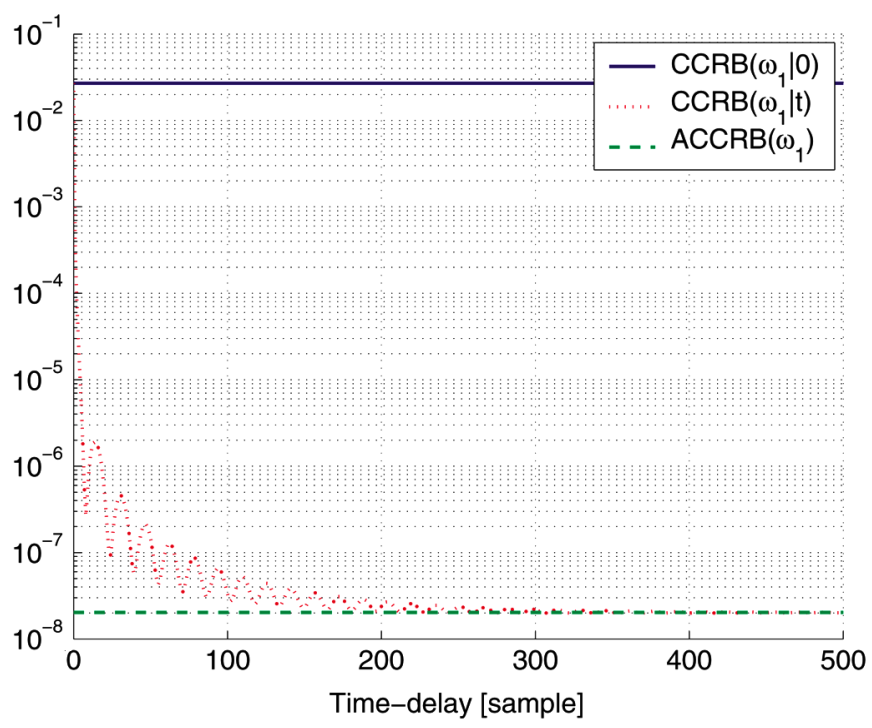

Fig. 1. $N=10^{4}$ samples, $\sigma^{2}=0.02, \quad \tau_{1,2}=t_{2} \in[0: 500]\left(t_{1}=0\right)$, $a=\left[\begin{array}{ll}1 & 1\end{array}\right]$, and $\phi=\left[\begin{array}{ll}\pi / 3 & 0\end{array}\right]$. (a) Well-separated poles: $\omega=\left[\begin{array}{ll}0.4 & 1\end{array}\right]$ and $|d|=[0.0040 .01]$. (b) Closely spaced poles: $\omega=\left[\begin{array}{ll}0.4 & 0.40001]\end{array}\right]$ and $|d|=$ $\left[\begin{array}{ll}0.01 & 0.01001\end{array}\right]$

\section{A. CRB Expression for the EDS Model}

The CRB for the EDS model can be derived from the CCRB since we have

$$
\begin{aligned}
\operatorname{CRB}(\Omega) & =\operatorname{CCRB}(\Omega \mid 0) \\
& =\frac{\sigma^{2}}{2} F_{0}^{-1}=\left(I_{2} \otimes A^{-1}\right) Q_{0}^{-1}\left(I_{2} \otimes A^{-1}\right)
\end{aligned}
$$

where

$$
Q_{0}=\mathfrak{R} e\left\{\left[\begin{array}{cc}
C & i C \\
-i C & C
\end{array}\right]\right\}
$$

with $c_{i j}=\xi_{i j}^{(1)} \xi_{i j}^{(2)} e^{i\left(\phi_{i}-\phi_{j}\right)}$ in the asymptotic context (i.e., $\left.N \rightarrow \infty\right)$.

\section{B. Strong Damping Factors}

We consider the case where the damping factors are strong and satisfy $\left|\tau_{i j} e^{d_{i} \tau_{i j}}\right| \ll 1$. In that case, the components are well separated in time and we can see from (15) that $\left|g_{i j}^{(\infty)}\right| \ll 1$ for $i \neq j$ due 
(a)

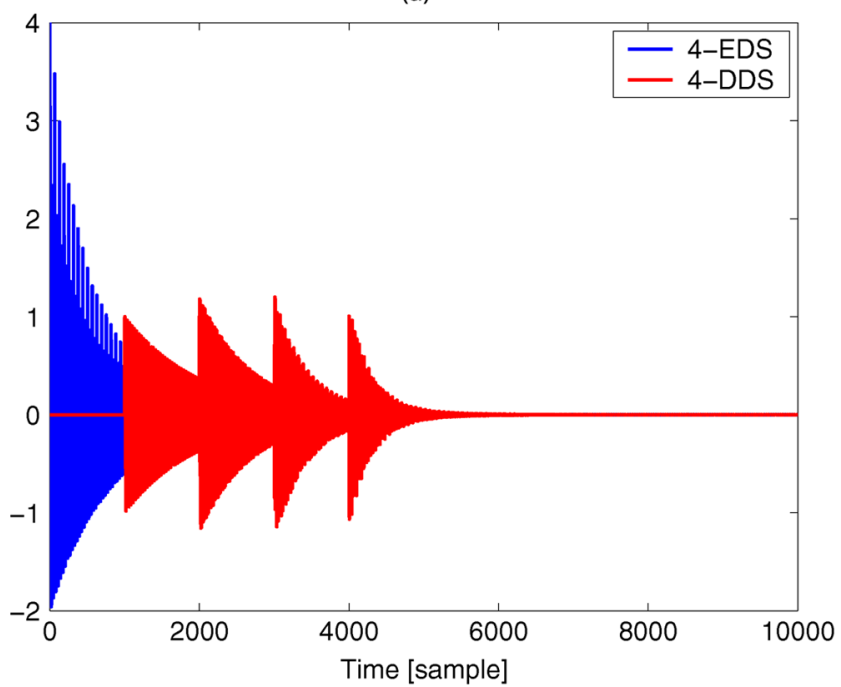

(b)

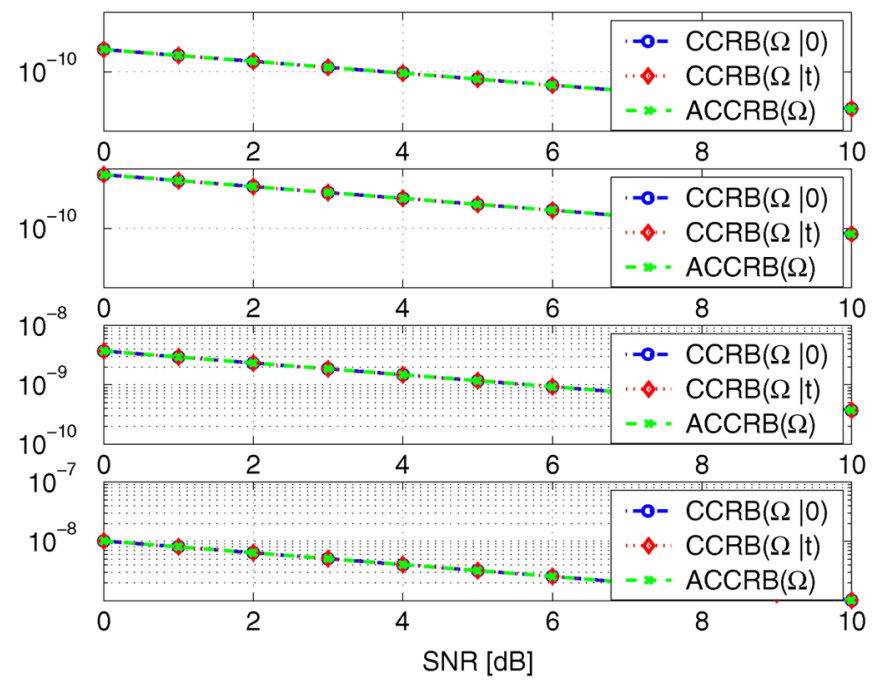

Fig. 2. (a) Time-domain signal with $\omega=\left[\begin{array}{lll}0.2 & 0.6 & 0.91 .1\end{array}\right], t=$ $\left[\begin{array}{llll}1000 & 2000 & 3000 & 4000\end{array}\right]$ and $|d|=\left[\begin{array}{llll}0.001 & 0.0015 & 0.0025 & 0.0035\end{array}\right]$. (b) CRBs for the EDS and DDS models.

to the exponential term $z_{i}^{\tau_{i j}}$. Hence, the FIM is again close to diagonal. Note that the asymptotic expression of the diagonal terms of the FIM is the same for the EDS and DDS models meaning that the strong damping-factor case corresponds to the situation of multiple 1-EDS models; so, the estimation of a given component is completely decorrelated from that of the others due to their separation in the time domain.

\section{Well-Separated Angular Frequencies and Low Damping Factors}

For well-separated angular frequencies with $\left|d_{i}\right| \ll 1$ for $i \in[1$ : $M]$, we have

$$
\begin{aligned}
\left\|F_{0}^{-1}-\mathbb{F}^{-1}\right\|^{2} & \leq \frac{\sigma^{4}}{4}\left\|I \otimes A^{-1}\right\|^{4}\left\|Q_{0}^{-1}-\mathbb{Q}^{-1}\right\|^{2} \\
& \leq \frac{\sigma^{4}}{4}\left\|I \otimes A^{-1}\right\|^{4}\left\|Q_{0}^{-1}\right\|^{2}\left\|\mathbb{Q}^{-1}\right\|^{2}\left\|Q_{0}-\mathbb{Q}\right\|^{2} \\
& =\rho\left\|Q_{0}^{-1}\right\|^{2}\|C-\mathbb{G}\|^{2}
\end{aligned}
$$

where $\rho=32 \sigma^{4}\left(\sum_{i=1}^{M}\left(1 / a_{i}^{2}\right)\right)^{2} \sum_{i=1}^{M} d_{i}^{6}$ is a strictly positive quantity. For well-separated sinusoids, $Q_{0}$ is a nonsingular matrix and thus (a)

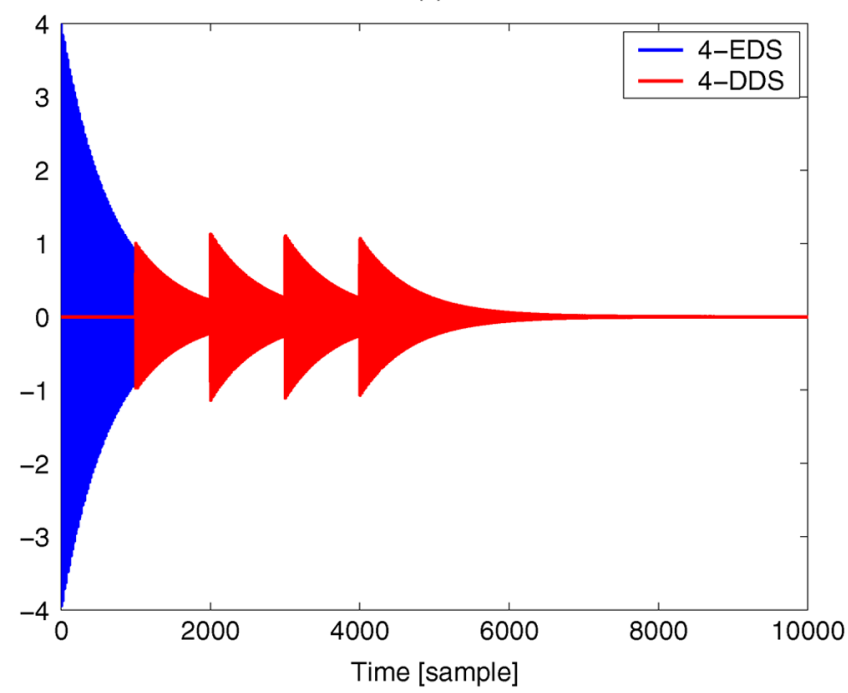

(b)

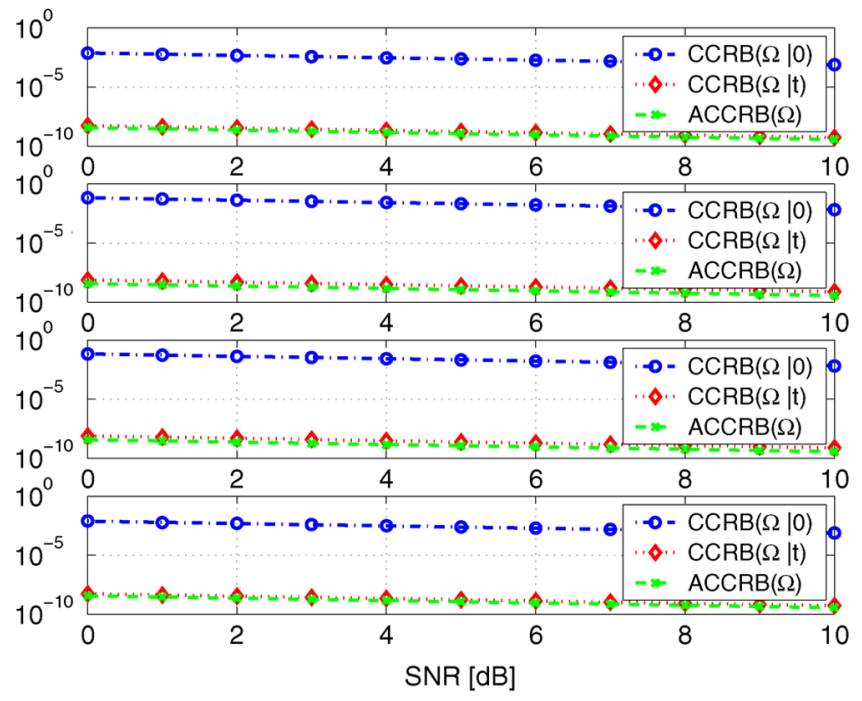

Fig. 3. (a) Time-domain signal with $\omega=\left[\begin{array}{lll}1 & 1.0001 & 1.0002\end{array}\right.$ $1.00031 .0004], t=\left[\begin{array}{llll}1000 & 2000 & 3000 & 4000\end{array}\right]$ and $|d|=$ $\left[\begin{array}{lllll}0.0015 & 0.001501 & 0.001502 & 0.001503 & 0.00105\end{array}\right]$. (b) CRBs for the EDS and DDS models.

$\left\|Q_{0}^{-1}\right\|<\infty$. In addition, we have

$$
\|C-\mathbb{G}\|^{2}=\sum_{i \neq j}\left|c_{i j}\right|^{2}=\frac{1}{4} \sum_{i \neq j} \frac{1+\cos \left(\omega_{i j}\right)}{\left(1-\cos \left(\omega_{i j}\right)\right)^{3}} .
$$

Expression (20) is a sum of positive terms that are, for well-separated angular frequencies, small compared to $\rho$. Indeed, for low damping factors, $\sum_{i=1}^{M} d_{i}^{6}$ is a very small quantity. Note that large real amplitude strengthens $\rho$ in this way. Consequently, $\left\|F_{0}^{-1}-\mathbb{F}^{-1}\right\|$ takes very small values and the CRB for the EDS is close to the ACCRB for the DDS model.

\section{Simulations}

\section{A. Comment on Assumption A3)}

Assumption A3), i.e., $\tau_{i, i+1} \gg 1$, is in practice not restrictive since $\tau_{i, i+1}$ can take relatively small values if the poles are far located [see Fig. 1(a)]. In the case of closely spaced poles, $\tau_{i, i+1}$ can take small 
values (typically few samples) to get the improvement of the CRB [as illustrated in Fig. 1(b)] but it should have moderate or large values for the approximate CRB to fit with the exact one [see Fig. 1(b)].

\section{B. Numerical CRBS}

In this part, we consider a 4-DDS model with unit amplitude and phase $\phi=[\pi / 3 \quad 0 \pi / 40]$. The analysis duration $N$ is $10^{4}$ samples. In Fig. 2, we have reported the considered signal and the CRBs for parameter $\Omega$. Note the good fit between the $\operatorname{ACCRB}(\Omega)$ and the $\operatorname{CCRB}(\Omega \mid t)$. In this situation, i.e., for well-separated sinusoids, the asymptotic performance for the DDS model is similar to that of the EDS model and the dependence with respect to the time-delay parameter can be neglected.

In Fig. 3, we consider closely spaced sinusoids. In this situation, we can note that the $\operatorname{ACCRB}(\Omega)$ and the $\operatorname{CCRB}(\Omega \mid t)$ are again very close and have not been affected by the closeness of the four poles. On the other hand, the CRB for the EDS model is much higher as expected. Consequently, the time-separation of the different DDS waveform allows the resolution of closely spaced sinusoids.

\section{CONCLUSION}

In this correspondence, we have derived the CRB for the DDS model. More precisely, we consider that the set of time delay is known and we name this bound the CCRB. We present a work out of the asymptotic CCRB corresponding to the scenarios of well-separated poles and closely (or even identical) poles. It is shown that in the former case 1) the ACCRB is close to that of the EDS model, 2) it depends only on the amplitudes and damping factors, and 3) the pole parameters are asymptotically decorrelated (i.e., the limit performance coincides with that of 1-EDS model). For closely spaced sinusoids, it is shown that the FIM is well conditioned in the DDS case while it becomes close to singular in the EDS case. Importantly, this is true not only in the trivial case of the well time-separated waveforms but also for overlapping waveforms with different time delays.

\section{ACKNOWLEDGMENT}

The authors would like to thank Prof. L. Tong and the reviewers for their valuable comments that led to the improvement of this correspondence.

\section{REFERENCES}

[1] Y. Hua and T. K. Sarkar, "Matrix pencil method for estimating parameters of exponentially damped/undamped sinusoids in noise," IEEE Trans. Acoust., Speech Signal Process., vol. 38, no. 5, pp. 814-824, May 1990.

[2] A. J. van der Veen, M. C. Vanderveen, and A. Paulraj, "Joint angle and delay estimation using shift-invariance techniques," IEEE Trans. Signal Process., vol. 46, no. 2, pp. 405-418, Feb. 1998

[3] R. Boyer and K. Abed-Meraim, "Damped and delayed model for transient modeling," IEEE Trans. Signal Process., vol. 53, no. 5, pp. 1720-1730, May 2005.

[4] S. Van Huffel, C. Decanniere, H. Chen, and P. Van Hecke, "Algorithm for time-domain NMR data fitting based on total least squares," J. Magn. Resonance A, vol. 110, pp. 228-237, 1994.

[5] M. Goodwin, "Adaptive signal models: Theory, algorithms, and audio applications," Ph.D. dissertation, Univ. California, Berkeley, CA, 1997.

[6] J. Nieuwenhuijse, R. Heusdens, and E. F. Deprettere, "Robust exponential modeling of audio signal," in Proc. Int. Conf. Acoust., Speech Signal Process., 1998, vol. 6.

[7] T. Wigren and A. Nehorai, "Asymptotic Cramer-Rao bounds for estimation of the parameters of damped sine waves in noise," IEEE Trans. Signal Process., vol. 39, no. 4, pp. 1017-1020, Apr. 1991.

[8] Y. Ying-Xian and S. M. Pandit, "Cramer-Rao lower bounds for a damped sinusoidal process," IEEE Trans. Signal Process., vol. 43, no. 4, Apr. 1995.

\section{Minimum Variance Channel Estimation in MC-CDMA Systems: Bias Analysis and Cramér-Rao Bound}

\author{
Shahrokh Nayeb Nazar, Student Member, IEEE, and \\ Ioannis N. Psaromiligkos, Member, IEEE
}

\begin{abstract}
We present a comprehensive performance analysis of the minimum variance channel estimator for multicarrier code-division multiple access systems. We provide novel highly accurate closed form expressions for the bias due to the additive noise as well as the finite data record mean-square error of the channel estimates. In addition, we derive the corresponding Cramér-Rao bound that assumes the knowledge of only the spreading code of the desired user.
\end{abstract}

Index Terms-Bias, Cramér-Rao bound (CRB), mean-square error (MSE), minimum variance (MV) channel estimation, multicarrier code division multiple access (MC-CDMA).

\section{INTRODUCTION}

Minimum variance (MV) algorithms are among the most popular methods for channel estimation and linear detection [1]-[4]. When employed for blind channel estimation, MV methods are particularly attractive compared to other second-order statistics (SOS)-based estimators, such as subspace MUSIC-type estimators [5], as they do not require rank estimation and show robustness to channel order overestimation [2]. MV methods require the knowledge of the auto-correlation matrix of the received data vectors that, in practice, is estimated from a data record of finite size. In this case, it is well known that the performance of the MV channel estimator is affected by two main factors. The first is finite sample effects, while the second is the additive noise which is the reason behind the fact that the MV estimator is asymptotically biased (as the sample size increases to infinity). A detailed study on the effect of noise on the asymptotic performance of the MV channel estimator for direct-sequence (DS)/CDMA systems has been performed in [1] under a small noise assumption. The theoretical analysis presented in [1] uses the spectral components of the received signal to derive a closed form expression for the asymptotic bias of the MV estimator. However, as we will illustrate, this approach does not produce accurate results for heavy system loads, small processing gains and/or severe multipath distortion.

In an attempt to circumvent these limitations, we derive in this correspondence a new expression for the asymptotic bias of the MV estimator for the downlink of multicarrier code-division multiple access (MC-CDMA) systems. Our analysis provides a close approximation to the channel estimation bias caused by the additive noise for MC-CDMA systems regardless of the system loading, channel length or processing gain. In addition, combining the derived approximation with the results of [2], we obtain the overall mean-square error (MSE) of the MV estimator for the case of a finite data record size. Finally, to benchmark the accuracy of the MV estimation algorithms we also derive the Cramér-Rao bound (CRB) for the (biased) MV estimator

Manuscript received March 3, 2006; revised September 7, 2006. This work was supported in part by the Natural Sciences and Engineering Research Council of Canada (NSERC) and in part by Le Fonds Quebecois de la Recherche sur la Nature et les Technologies. The associate editor coordinating the review of this manuscript and approving it for publication was Dr. Athanasios P. Liavas.

The authors are with the Department of Electrical and Computer Engineering, McGill University, Montréal, QC H3A-2A7 Canada (e-mail: shahrokh.nayebnazar@mail.mcgill.ca; yannis@ece.mcgill.ca).

Digital Object Identifier 10.1109/TSP.2007.893975 